\title{
Hadrontherapy: the ENLIGHT network
}

Manjit Dosanjh ${ }^{1}$, Audrey Ballantine ${ }^{1}$, Manuela Cirilli ${ }^{1}$, Helen Dixon-Altaber ${ }^{1}$, Virginia Greco ${ }^{1,2}$

${ }^{1}$ CERN (Switzerland), ${ }^{2}$ University of Siena (Italy)

The use of ionizing radiation as a treatment for cancer is nowadays well established, but the traditional radiotherapy, which employs very energetic photons (X-rays), has several side effects. Some dose is deposited in healthy tissues so that the toxicity of the treatment is quite high.

Hadrontherapy is a new generation of radiotherapy, based on the use of charged particles (protons and ions), which can penetrate the tissue with little diffusion and deposit the maximum energy in a defined small area, so that important orgasn and healthy tissues can be spared more easily .

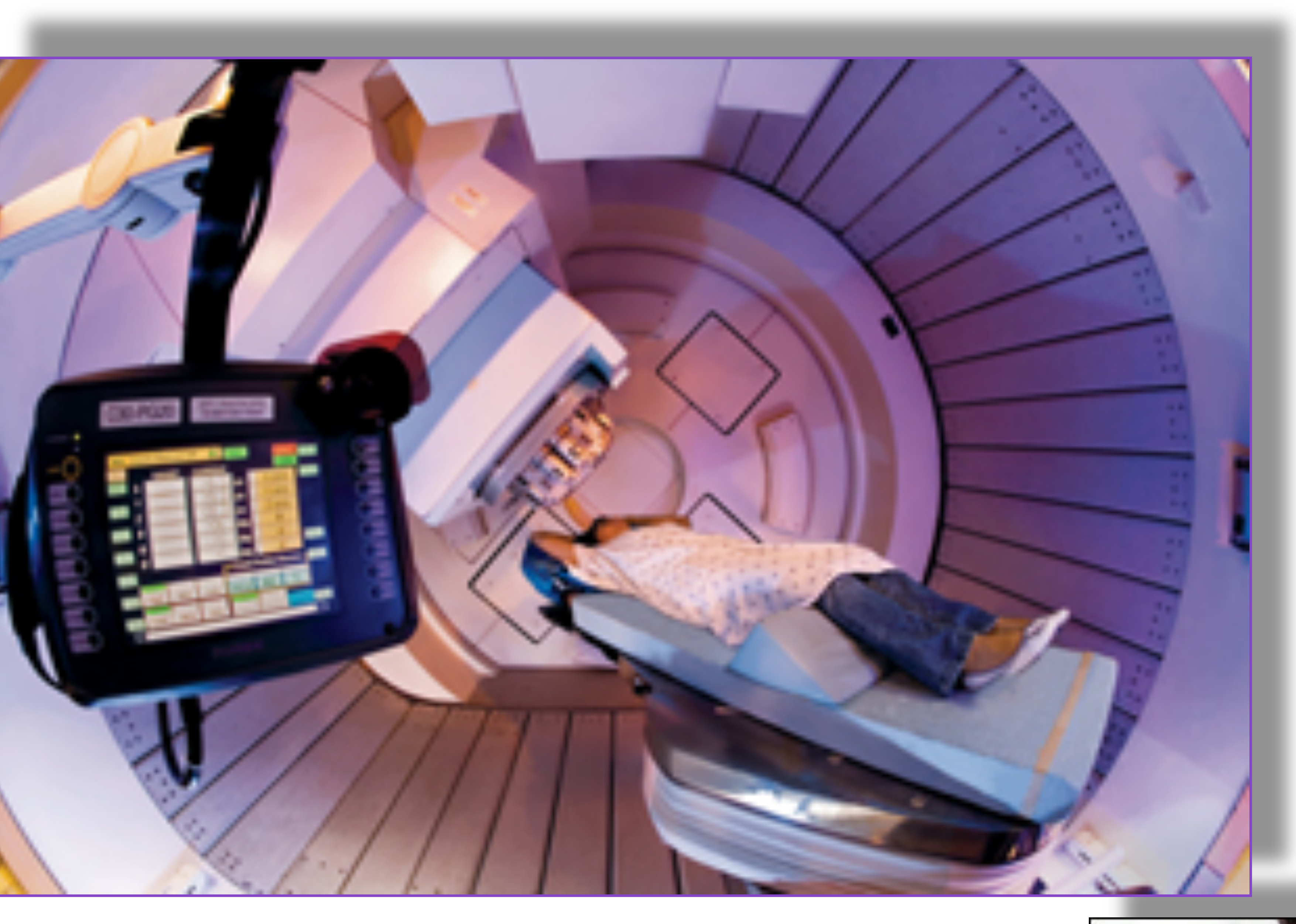

The ENLIGHT network:

$\Lambda$ gathers experts who work in the same field but come from different backgrounds:

$\rightarrow$ physicists, physicians, biologists, engineers; $\iota$ promotes the sharing of knowledge and expertise; $\iota$ provides training for young researchers;

$\iota$ identifies challenges;

$\iota$ implements actions to meet future requirements in hadrontherapy;

$\iota$ develops strategies to secure the funding for future projects.

ENLIGHT, the European Network for Light Ion Hadron Therapy:

$\Lambda$ was established in 2002;

$\Lambda$ involves more than 300 participants from 20 European countries;

$\Lambda$ connect European universities, institutions and industries involved in research on hadron-therapy
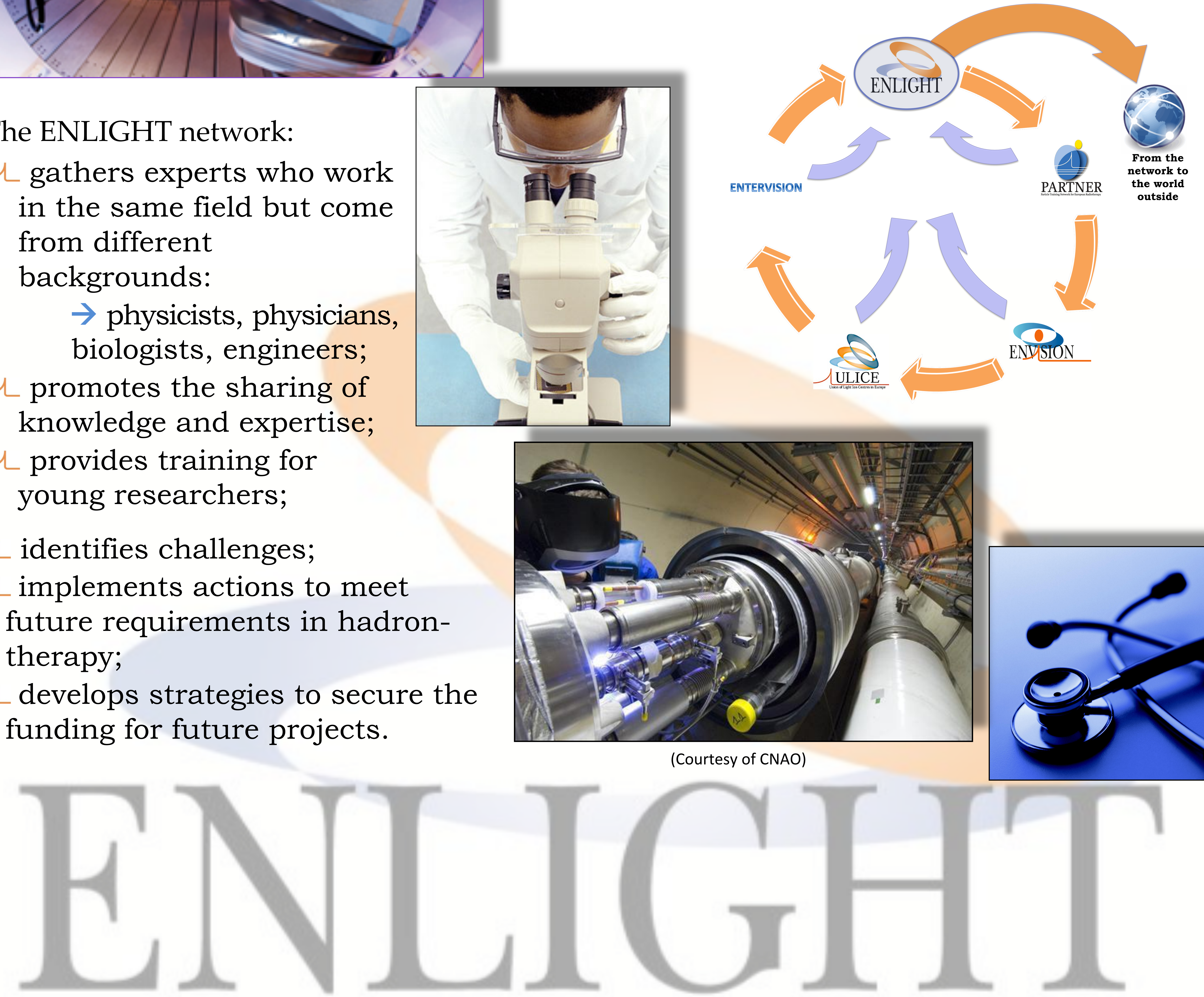

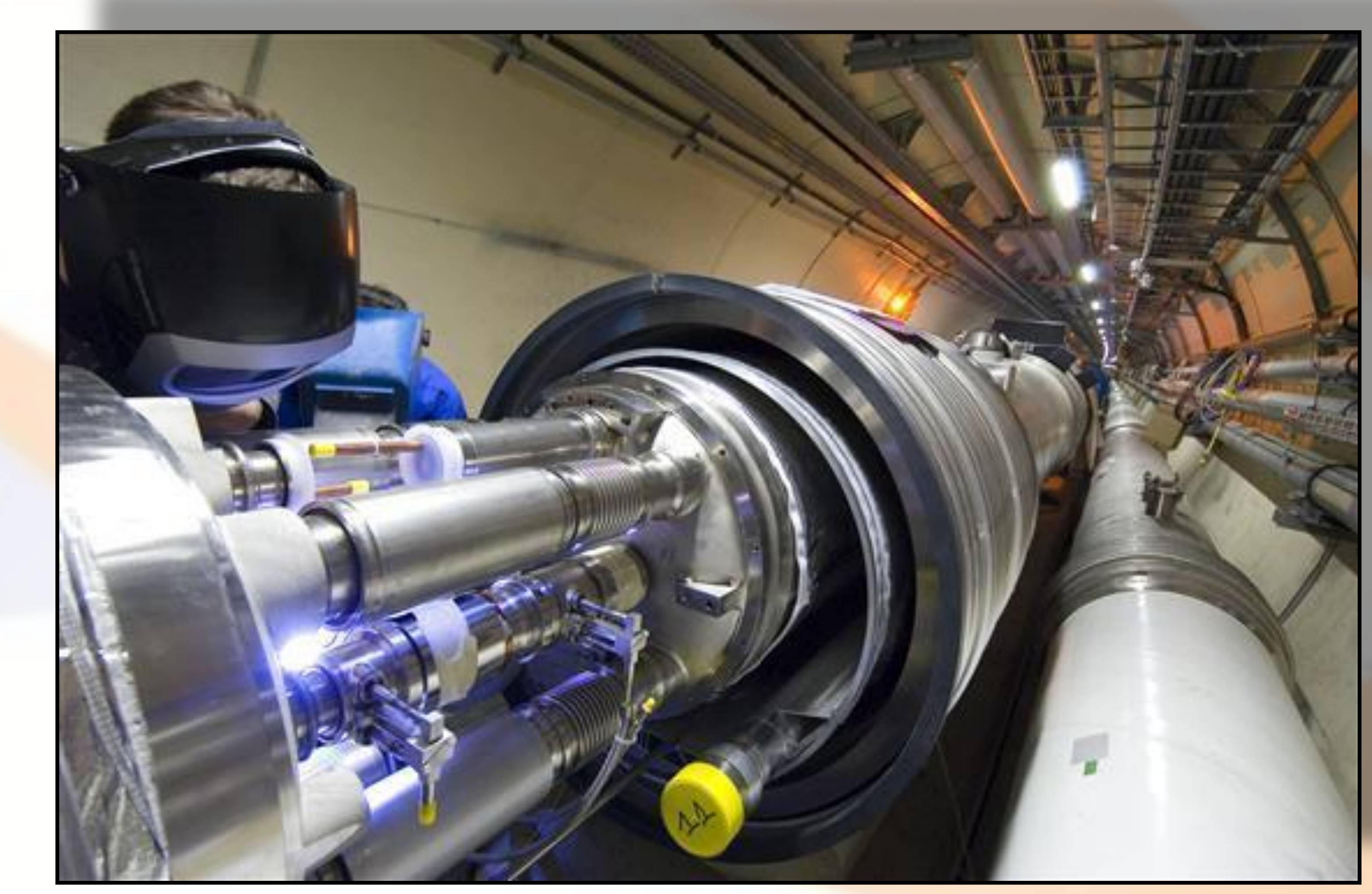

(Courtesy of CNAO)

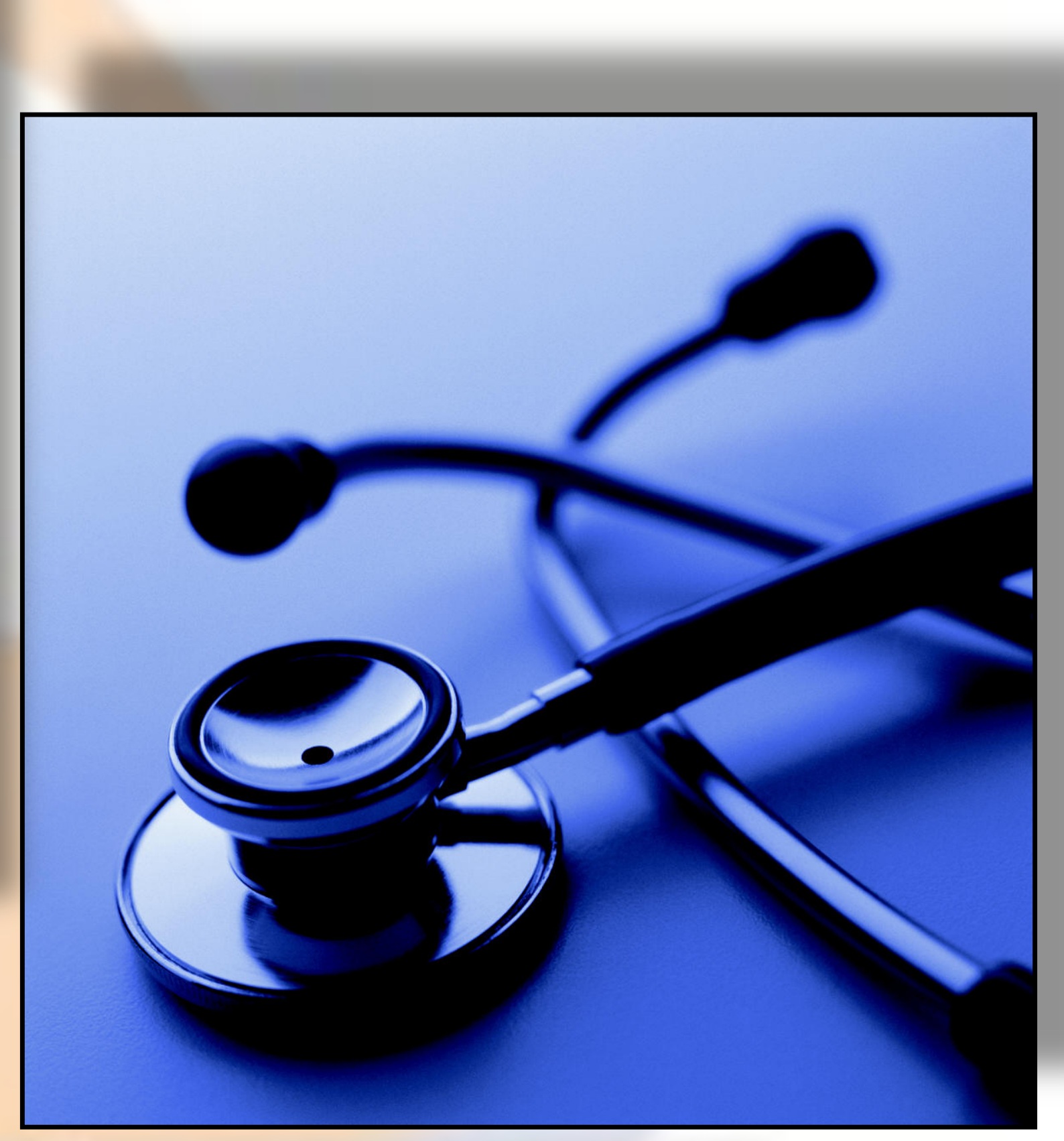

